Canadian

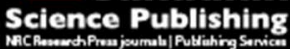

Applied Physiology, Nutrition, and Metabolism Physiologie appliquée, nutrition et métabolisme

\title{
Effect of electrical stimulation-induced resistance exercise on mitochondrial fission and fusion proteins in rat skeletal muscle
}

\begin{tabular}{|r|l|}
\hline Journal: & Applied Physiology, Nutrition, and Metabolism \\
\hline Manuscript ID: & apnm-2015-0184.R2 \\
\hline Manuscript Type: & Article \\
\hline Date Submitted by the Author: & 08-Jul-2015 \\
\hline Complete List of Authors: & $\begin{array}{l}\text { Kitaoka, Yu; The University of Tokyo, Department of Sports Sciences } \\
\text { Ogasawara, Riki; The University of Tokyo, Department of Sports Sciences } \\
\text { Tamura, Yuki; The University of Tokyo, Department of Sports Sciences } \\
\text { Fujita, Satoshi; Ritsumeikan University, Faculty of Sport and Health } \\
\text { Science } \\
\text { HATTA, HIDEO; University of Tokyo }\end{array}$ \\
\hline Keyword: & $\begin{array}{l}\text { resistance exercise < exercise, exercise metabolism < exercise } \\
\text { metabolism, skeletal muscle < skeletal muscle }\end{array}$ \\
\hline \multicolumn{2}{|c}{} \\
\hline
\end{tabular}

\section{SCHOLARONE}

Manuscripts 
1 Effect of electrical stimulation-induced resistance exercise on mitochondrial fission and

\section{2 fusion proteins in rat skeletal muscle}

3

4 Yu Kitaoka ${ }^{1}$, Riki Ogasawara ${ }^{1}$, Yuki Tamura ${ }^{1}$, Satoshi Fujita ${ }^{2}$, and Hideo Hatta ${ }^{1}$

5

6

$7 \quad{ }^{1}$ Department of Sports Sciences, Graduate School of Arts and Sciences, The University of

8 Tokyo, Tokyo, Japan

$9 \quad{ }^{2}$ Faculty of Sport and Health Science, Ritsumeikan University, Kusatsu, Shiga, Japan

10

11

12 Running Head: Resistance exercise and mitochondrial dynamics

13

14

15

16

Correspondence: Yu Kitaoka

17

Department of Sports Sciences, Graduate School of Arts and Sciences, The University of Tokyo,

18 3-8-1 Komaba, Meguro-ku, Tokyo 153-8902, Japan

19 Tel: +81-3-5454-6858; Fax: +81-3-5454-4317; E-mail: kitaoka@idaten.c.u-tokyo.ac.jp

20

$21 \quad$ E-mail addresses

22 YK (kitaoka@idaten.c.u-tokyo.ac.jp), RO (ogasawara@idaten.c.u-tokyo.ac.jp),

23 YT (9696053430@mail.ecc.u-tokyo.ac.jp), SF (safujita@fc.ritsumei.ac.jp),

24 HH (hatta@idaten.c.u-tokyo.ac.jp)

25

26 


\section{$27 \quad$ ABSTRACT}

28 It is well known that resistance exercise increases muscle protein synthesis and muscle strength.

29 However, little is known about the effect of resistance exercise on mitochondrial dynamics, 30 which is coupled with mitochondrial function. In skeletal muscle, mitochondria exist as 31 dynamic networks that are continuously remodeling through fusion and fission. The purpose of 32 this study was to investigate the effect of acute and chronic resistance exercise, which induces 33 muscle hypertrophy, on the expression of proteins related to mitochondrial dynamics in rat 34 skeletal muscle. Resistance exercise consisted of maximum isometric contraction, which was 35 induced by percutaneous electrical stimulation of the gastrocnemius muscle. Our results 36 revealed no change in mitochondrial fission (Fis1 and Drp1) or fusion (Opa1, Mfn1, and Mfn2) 37 regulatory protein levels over the 24-h period following acute resistance exercise. 38 Phosphorylation of Drp1 at Ser616 was increased immediately after exercise $(P<0.01)$. Four 39 weeks of resistance training (three times/week) increased Mfn1 $(P<0.01)$, Mfn2 $(P<0.05)$, and 40 Opa1 $(P<0.01)$ protein levels without altering mitochondrial oxidative phosphorylation proteins.

41 These observations suggest that resistance exercise has little effect on mitochondrial biogenesis, 42 but alters the expression of proteins involved in mitochondrial fusion and fission, which may 43 contribute to mitochondrial quality control and improved mitochondrial function.

45 Key Words: skeletal muscle; resistance exercise; mitochondrial dynamics; Fis1; Drp1; Opa1; 46 Mfn 


\section{INTRODUCTION}

54 It has been well recognized that resistance exercise increases muscle protein synthesis and 55 muscle strength, and thus can delay age-associated declines in muscle mass and function. 56 However, the impact of resistance exercise on energy metabolism in skeletal muscle remains 57 relatively unknown. Previous studies have shown that resistance exercise largely does not alter 58 the maximal activity of mitochondrial enzymes such as citrate synthase and succinate 59 dehydrogenase, which are considered to reflect mitochondrial volume (Tesch et al. 1990; Green

60 et al. 1999b). More recent studies have found mitochondrial respiration to increase after 61 resistance training in human skeletal muscle (Pesta et al. 2011; Salvadego et al. 2013; Porter et 62 al. 2014). Therefore, resistance exercise training may increase mitochondrial function without 63 changes in mitochondrial mass.

64

65 In skeletal muscle, mitochondria exist as dynamic networks that are continuously remodeling 66 through fusion and fission. These processes, termed mitochondrial dynamics, are important for 67 maintenance of functional mitochondria. Mitochondrial fusion is regulated by mitofusin 68 proteins (Mfn1 and Mfn2) and by optic atrophy 1 (Opa1) in the outer and inner mitochondrial 69 membrane, respectively (Meeusen et al. 2006; Chen et al. 2010). Opa1 undergoes proteolytic 70 processing, which has been shown to be essential in fusion activity (Duvezin-Caubet et al. 2006;

71 Wang et al. 2014). Mitochondrial fission is mediated by dynamin related protein 1 (Drp1), a 72 cytosolic protein that translocates to the outer surface of the mitochondria when activated, in 73 combination with fission protein 1 (Fis1) (Romanello et al. 2010). Drp1 is activated by the 74 phosphorylation of serine 616 (Taguchi et al. 2007). Disorders of mitochondrial dynamics can 75 lead to reduced mitochondrial respiratory capacity (Bach et al. 2003; Chen et al. 2005; Parone et 76 al. 2008), suggesting that balanced mitochondrial fisson and fusion events are critical to the 77 maintenance of mitochondrial function. 
79 Dysfunction of skeletal muscle mitochondria in type 2 diabetes or chronic muscle disuse has 80 been reported to be associated with a decrease in the abundance of mitochondrial fusion 81 proteins or with an increase in the activation status of the mitochondrial fission protein Drp1 82 (Bach et al. 2003; Iqbal et al. 2013; Picard et al. 2015). In contrast, a recent study demonstrated 83 that an endurance exercise-type chronic muscle contractile activity increased mitochondrial 84 fusion protein levels (Iqbal et al. 2013). Moreover, exercise training decreases the activation 85 status of Drp1 in insulin-resistant human skeletal muscle (Fealy et al. 2014). Therefore, the 86 increase in skeletal muscle oxidative capacity with exercise training may not only attribute to 87 mitochondrial biogenesis, but also to changes in mitochondrial dynamics. However, the effects 88 of resistance exercise on mitochondrial dynamics proteins have not yet been examined. The 89 purpose of this study was to examine the abundance and activation status of the mitochondrial 90 fission (Fis1 and Drp1) and fusion (Opa1 and Mfn2) regulatory proteins following acute and 91 chronic resistance exercise in rat skeletal muscle.

\section{MATERIALS AND METHODS}

\section{Animals}

95 Thirty-five male Sprague-Dawley rats (male, aged 10 weeks) were obtained from CLEA Japan 96 (Tokyo, Japan). All animals were housed individually in an environment maintained at 22-24

97 with a 12-h light-dark cycle and were allowed food and water ad libitum. Rats were randomly 98 assigned to one of seven groups: sedentary (SED), acute resistance exercise (RE), acute 99 resistance exercise followed by $1 \mathrm{~h}$ of recovery $(\mathrm{RE}+1 \mathrm{~h})$, as well as $3 \mathrm{~h}(\mathrm{RE}+3 \mathrm{~h}), 6 \mathrm{~h}(\mathrm{RE}+6 \mathrm{~h})$, 100 and $24 \mathrm{~h}(\mathrm{RE}+24 \mathrm{~h})$, and four weeks of resistance exercise training (RT). This study was

101 approved by the Ethics Committee for Animal Experiments at Ritsumeikan University.

\section{Experimental protocol}

103 Under isoflurane anesthesia, the hair was shaved off the right lower leg of each rat, and then the 104 rats were positioned with their right foot on a footplate (the ankle joint angle was positioned at 
$1059^{\circ}$ ) in the prone posture. The triceps surae muscle was stimulated percutaneously with with 106 electrodes (Vitrode $\mathrm{V}, \mathrm{Ag} / \mathrm{AgCl}$; Nihon Kohden, Tokyo, Japan), which were cut into $10 \times 5-\mathrm{mm}$ 107 sections and connected to an electric stimulator and isolator (SS-104J; Nihon Kohden, Japan). 108 The right gastrocnemius muscle was isometrically exercised as previously described 109 (Ogasawara et al. 2013; Ogasawara et al. 2014). For all exercise sessions, the gastrocnemius 110 muscle was trained by stimulating ten 3-s contractions, with a 7-s interval between contractions,

111 per set for 5 sets, with 3-min rest intervals. The voltage $(\sim 30 \mathrm{~V})$ and stimulation frequency (100 $112 \mathrm{~Hz}$ ) were adjusted to produce maximal isometric tension. Animals were euthanized and the 113 target tissues were removed at a basal state, and at immediately, $1 \mathrm{~h}, 3 \mathrm{~h}, 6 \mathrm{~h}, 24 \mathrm{~h}$ after the 114 completion of exercise. Rats in the RT group were trained every other day for 4 weeks (12 115 exercise sessions in total) using the same electrical stimulation protocol described above, and 116 the left gastrocnemius muscle served as an internal control for the examination of training 117 adaptations. Animals were euthanized and the target tissues were removed $48 \mathrm{~h}$ after the last 118 exercise session. The tissues were rapidly frozen in liquid nitrogen and stored at $-80 \square$ until 119 further analysis.

\section{Western blotting}

121 Whole gastrocnemius muscle samples were homogenized using a Polytron homogenizer in 122 homogenization buffer $(20 \mathrm{mM}$ Tris- $\mathrm{HCl}, \mathrm{pH} 7.5,150 \mathrm{mM} \mathrm{NaCl}, 1 \% \mathrm{NP}-40,1 \%$ sodium 123 deoxycholate, $1 \mathrm{mM}$ EDTA and $0.1 \%$ SDS) supplemented with protease and phosphatase 124 inhibitor cocktail (Thermo Fisher Scientific, Waltham, MA). The homogenates were centrifuged 125 at $10,000 \mathrm{~g}$ for $10 \mathrm{~min}$ at $4^{\circ} \mathrm{C}$. The supernatant was removed, and the protein concentration of 126 each sample was quantified using a Protein Assay Rapid Kit (Wako, Japan). Equal amounts of 127 protein $(5-15 \mu \mathrm{g}$, depending on the protein of interest) were loaded onto 10-12\% SDS-PAGE 128 gels and separated by electrophoresis. Proteins were transferred to polyvinylidene difluoride 129 membranes, and Western blotting was carried out using the primary antibodies: Total OXPHOS 130 Rodent WB Antibody Cocktail [NDUFB8 (NADH dehydrogenase (ubiquinone) 1 beta 
131 subcomplex 8), SDHB (succinate dehydrogenase complex subunit B), UQCRC2

132 (ubiquinol-cytochrome c reductase core protein II), ATP5A (ATP synthase, H+ transporting, 133 mitochondrial F1 complex, alpha subunit 1); ab110413], LDH (lactate dehydrogenase; 134 ab134187), FAT/CD36 (fatty acid translocase; ab137320), Fis1 (ab96764), Drp1 (ab56788), 135 Mfn2 (ab124773), and Parkin (ab77924) from Abcam (Cambridge, MA); COX IV (cytochrome 136 c oxidase subunit 4; \#4850), SOD2 (Superoxide dismutase 2; \#13141), and Phospho-Drp1 137 (Ser616, \#3455) from Cell Signaling Technology (Danvers, MA); Opa1 (\#612606) from BD 138 transduction laboratories (Tokyo, Japan); Mfn1 (sc-50330) and PFK (phosphofructokinase; 139 sc-31712) from Santa Cruz Biotechnology (Santa Cruz, CA); GLUT4 (\#07-1404) from 140 Millipore (Billerica, MA). Antibodies against MCT (monocarboxylate transporter)1 and MCT4 141 were raised in rabbits against the C-terminal region of the respective MCT (Qiagen, Japan) and 142 have been used in previous studies (Enoki et al. 2006; Kitaoka et al. 2014). Membranes were 143 then incubated with the appropriate secondary antibody (A106PU or A102PT; American Qualex, 144 San Clemente, CA or sc-2020; Santa Cruz Biotechnology) and visualized by enhanced 145 chemiluminescence detection reagent (Thermo Fisher Scientific). Blots were scanned and 146 quantified using ChemiDoc XRS (Bio-Rad Laboratories, Hercules, CA) and Quantity One 147 (version 4.5.2, Bio-Rad). Ponceau staining was used to verify consistent loading.

\section{Statistical analysis}

149 Data were expressed as mean \pm SEM. Multi-group comparisons in acute study were performed 150 by one-way analysis of variance (ANOVA), followed by the Dunnett post hoc test. Paired t-tests 151 were used to test for differences between groups in chronic study (GraphPad Prism 5.0, La Jolla, 152 CA). Values of $P<0.05$ were considered significant.

\section{RESULTS}

155 Effect of acute resistance exercise on mitochondrial dynamics proteins

156 To examine the adaptations of mitochondrial fusion and fission proteins to resistance exercise, 
157 we first investigated the time course of protein expression after acute exercise. We did not 158 identify any changes in the level of mitochondrial fission protein Fis1. Phosphorylation of Drp1 159 at Ser616 was increased immediately after exercise $(+137 \%, P<0.01)$ and 1 hour after exercise $160(+75 \%, P<0.05)$, without changes in total Drp1 protein abundance. There was no change in the 161 level of mitochondrial fusion proteins Mfn1, Mfn2, or total Opa1 over the 24-h period following 162 acute resistance exercise. As it has been previously reported, knockdown of Opa1-induced 163 mitochondrial fragmentation is recovered by the expression of the long isoform of Opa1 164 (L-Opa1) but not the short isoform of Opa1 (S-Opa1) (Ishihara et al. 2006), which indicates that 165 L-Opa1 is the active isoform. We quantified these isoforms separately, but did not find any 166 changes in either isoform of Opa1 or the ratio of L-Opa1 to S-Opa1 (Figure 1).

\section{Effect of chronic resistance training on mitochondrial dynamics proteins}

168 Next, we examined the effect of chronic resistance exercise on the levels of mitochondrial 169 dynamics proteins. Four weeks of resistance training (12 exercise sessions) increased Mfn1 $170(+24 \%, P<0.01)$ and Mfn2 $(+14 \%, P<0.05)$ protein levels. Both $\mathrm{S}-$ and L-isoforms of Opa1 and 171 total Opa1 levels were also increased $(+20 \%, P<0.01)$. There were no changes in the levels of 172 Fis1, total Drp1, or Drp1 phosphorylation at Ser616 (Figure 2).

\section{Effect of chronic resistance training on energy metabolism proteins}

174 Mitochondrial oxidative phosphorylation (OXPHOS) protein levels were not altered by 4 weeks 175 of resistance training, except for UQCRC (complex $\square$ ), which slightly increased $(+8 \%, P<0.05)$. 176 Levels of superoxide dismutase 2 (SOD2), a mitochondrial specific antioxidant enzyme, 177 increased after resistance training $(+11 \%, P<0.05)$. We did not observe any changes in Parkin, a 178 protein involved in mitophagy (Figure 3A), coinciding with a recent human study (Ogborn et al. 179 2015). Glycolytic enzyme protein levels (phosphofructokinase and lactate dehydrogenase) were 180 not altered by resistance training (Figure 3B). Levels of glucose, lactate, and fatty acid 181 transporter proteins did not change after resistance training, except for MCT4, which markedly 182 increased $(+24 \%, P<0.05)$ (Figure 3C). 


\section{DISCUSSION}

185 To the best of our knowledge, changes in the abundance and activation status of mitochondrial 186 fusion and fission regulatory proteins by resistance exercise have not been investigated in 187 skeletal muscle. In this study, we used the rat electrical stimulation model and have shown that

188 1) acute resistance exercise increased the activation status of Drp1 and 2) chronic resistance 189 training increased mitochondrial fusion protein Mfn1, Mfn2, and Opa1 levels without altering 190 mitochondrial mass in rat skeletal muscle. In our previous studies, we demonstrated that this 191 exercise protocol induces mammalian target of rapamycin (mTOR) signaling activation, 192 associated with increased muscle protein synthesis, and that long-term training induces 193 significant increases in muscle size and strength (Ogasawara et al. 2013; Ogasawara et al. 2014).

194 To further validate our rat isometric training model, we examined proteins involved in 195 glycolysis, OXPHOS, and metabolite transport. We observed no changes in most of these 196 proteins related to energy metabolism, which is consistent with previous human studies that 197 reported no changes in either glycolytic or oxidative enzyme activities after resistance training 198 (Tesch et al. 1990; Green et al. 1999a). This adaptation is clearly different from endurance 199 training or high-intensity interval training, which are known to upregulate metabolite transport 200 proteins for each specific substrate uptake, accompanied by mitochondrial biogenesis 201 (Burgomaster et al. 2008; Perry et al. 2008; Hoshino et al. 2013). In this study, we observed a 202 significant increase in MCT4, a protein that facilities lactate release from muscle cells. This 203 observation is consistent with a previous human study (Juel et al. 2004). Importantly, expression 204 of MCT1, but not MCT4, increased after 1 and 3 weeks of chronic muscle stimulation $(10 \mathrm{~Hz}$, $20524 \mathrm{~h} /$ day), an endurance exercise model in rats (Bonen et al. 2000). Thus, in this study we 206 utilized high-frequency muscle contractions as the model of resistance exercise in rats. Our 207 findings suggest that acute and chronic resistance exercise alters the activation status and 208 abundance of mitochondrial dynamics proteins without altering mitochondrial volume. 
210 Previous studies have demonstrated that acute exercise may potentially promote the 211 mitochondrial fission process. Treadmill running for $150 \mathrm{~min}$ reported to increase Fis1 protein 212 level and decrease Mfn1 protein in rat skeletal muscle (Ding et al. 2010). Another study has 213 shown that 90 min of treadmill running increased phosphorylation of Drp1 at Ser616 without 214 altering expression of Mfn1 and Mfn2 in skeletal muscle of mice (Jamart et al. 2013). In this 215 study, we found that the activation status of Drp1 was increased immediately after acute 216 resistance exercise. Recent studies have shown that Drp1 phosphorylation at Ser616 is regulated 217 by extracellular signal-regulated protein kinase (ERK)1/2 (Kashatus et al. 2015; Serasinghe et al. 218 2015). Interestingly, this pathway is well known to be activated by resistance exercise (Karlsson 219 et al. 2004; Moore et al. 2011). In addition, skeletal muscle contraction-induced reactive oxygen 220 species (ROS) production can induce mitochondrial fragmentation (Fan et al. 2010). Acute 221 exercise-activated fission process might be important in removing damaged mitochondria, since 222 autophagy is reported to be required for training adaptation in skeletal muscle (Lira et al. 2013).

223 In addition, fission may be conceivably required to remodel the mitochondrial network.

224 However, at present, very little information is available regarding changes in mitochondrial 225 morphology followed by acute exercise. One study has demonstrated that 3-h voluntary exercise 226 remodels mitochondrial membrane interactions, without changes in Mfn2 and Opa1 in mouse 227 skeletal muscle (Picard et al. 2013). Further studies are needed to understand the acute 228 exercise-induced mitochondrial remodeling.

230 In contrast to acute exercise, chronic exercise training may induce the mitochondrial fusion 231 process. A recent study demonstrated that 7 days of chronic muscle contractile activity $(10 \mathrm{~Hz}, 3$ $232 \mathrm{~h}$ /day), an endurance exercise model in rats, increased protein levels of Mfn2 and Opa1, while 233 Drp1 was decreased and Fis1 was not affected (Iqbal et al. 2013). In a human study, 2 weeks of 234 high-intensity interval training was shown to increase protein levels of Fis1, Drp1, and Mfn1, 
235 but not Mfn2 (Perry et al. 2010). Other studies in humans (Cartoni et al. 2005) and rats (Ding et

236 al. 2010) have also reported an increase in Mfn1 and Mfn2 transcript levels. However, transcript 237 and protein levels of mitochondrial dynamics proteins may not directly translate to altered 238 fission/fusion, given that these proteins are regulated by post-translational modifications and 239 proteolytic processing (Duvezin-Caubet et al. 2006; Ishihara et al. 2006; Taguchi et al. 2007).

240 Therefore, in the current study, we evaluated the phosphorylation of Drp1 at Ser616 and L-Opa1,

241 which represented the activation status of these proteins, in addition to their total protein 242 abundances. We found that 4 weeks of resistance training increased L-Opa1 protein level, while 243 phosphorylation of Drp1 at Ser616 was not altered in rat skeletal muscle. Protein levels of total 244 Opa1, Mfn1, and Mfn2 also increased, whereas total Drp1 and Fis1 levels were unchanged. Our 245 results suggest that chronic resistance training activates the mitochondrial fusion process, which

246 possibly leads to an expanded mitochondrial reticular network along with muscle hypertrophy,

247 although the absence of actual mitochondrial morphology data via electron microscopy is the 248 main limitation of this study. Nevertheless, previous studies which demonstrated that repression 249 of Mfn2 reduces respiratory capacity and mitochondrial membrane potential in muscle cells 250 (Bach et al. 2003), or loss of Mfn1 and Mfn2 causes severe mitochondrial dysfunction and 251 muscle atrophy with high levels of mtDNA mutations (Chen et al. 2010), support the 252 importance of increased mitochondrial fusion proteins in response to resistance training.

254 It was generally assumed that resistance training has little effect on skeletal muscle 255 mitochondria, since maximal activity of oxidative enzymes is not altered. In the current study, 256 we demonstrated that acute and chronic resistance exercise altered the activation status and total 257 protein abundance of proteins involved in mitochondrial fusion and fission, which may 258 contribute to improved mitochondrial and subsequently muscle function. 259

260 Conflict of interest statement: There is no conflict of interest. 
262 Acknowledgments: This study was supported by a grant-in-aid for young scientists (B; 26326750304 and A; 26702028) from the Japan Society for the Promotion of Science (JSPS) and 264 Yamaha Motor Foundation for Sports (FMFS).

\section{References}

267

Bach, D., Pich, S., Soriano, F.X., Vega, N., Baumgartner, B., Oriola, J., et al. 2003. Mitofusin-2 determines mitochondrial network architecture and mitochondrial metabolism. A novel regulatory mechanism altered in obesity. J Biol Chem 278: 17190-17197.

270 Bonen, A., Tonouchi, M., Miskovic, D., Heddle, C., Heikkila, J.J., and Halestrap, A.P. 2000.

271 Isoform-specific regulation of the lactate transporters MCT1 and MCT4 by contractile activity.

272 Am J Physiol Endocrinol Metab 279: E1131- E1138.

273 Burgomaster, K.A., Howarth, K.R., Phillips, S.M., Rakobowchuk, M., Macdonald, M.J., McGee, 274 S.L., et al. 2008. Similar metabolic adaptations during exercise after low volume sprint interval 275 and traditional endurance training in humans. J Physiol 586: 151-160.

276 Cartoni, R., Leger, B., Hock, M.B., Praz, M., Crettenand, A., Pich, S., et al. 2005. Mitofusins $277 \quad 1 / 2$ and ERRalpha expression are increased in human skeletal muscle after physical exercise. $\mathbf{J}$ 278 Physiol 567: 349-358.

279 Chen, H., Chomyn, A., and Chan, D.C. 2005. Disruption of fusion results in mitochondrial 280 heterogeneity and dysfunction. J Biol Chem 280: 26185-26192.

281 Chen, H., Vermulst, M., Wang, Y.E., Chomyn, A., Prolla, T.A., McCaffery, J.M., et al. 2010. 282 Mitochondrial fusion is required for mtDNA stability in skeletal muscle and tolerance of 283 mtDNA mutations. Cell 141: 280-289.

284 Ding, H., Jiang, N., Liu, H., Liu, X., Liu, D., Zhao, F., et al. 2010. Response of mitochondrial 285 fusion and fission protein gene expression to exercise in rat skeletal muscle. Biochim Biophys 286 Acta 1800: 250-256.

287 Duvezin-Caubet, S., Jagasia, R., Wagener, J., Hofmann, S., Trifunovic, A., Hansson, A., et al. 288 2006. Proteolytic processing of OPA1 links mitochondrial dysfunction to alterations in 289 mitochondrial morphology. J Biol Chem 281: 37972-37979.

290 Enoki, T., Yoshida, Y., Lally, J., Hatta, H., and Bonen, A. 2006. Testosterone increases lactate 291 transport, monocarboxylate transporter (MCT) 1 and MCT4 in rat skeletal muscle. J Physiol 
577: 433-443.

293 Fan, X., Hussien, R., and Brooks, G.A. 2010. H2O2-induced mitochondrial fragmentation in 294 C2C12 myocytes. Free Radic Biol Med 49: 1646-1654.

295 Fealy, C.E., Mulya, A., Lai, N., and Kirwan, J.P. 2014. Exercise training decreases activation of 296 the mitochondrial fission protein dynamin-related protein-1 in insulin-resistant human skeletal 297 muscle. J Appl Physiol (1985) 117: 239-245.

298 Green, H., Dahly, A., Shoemaker, K., Goreham, C., Bombardier, E., and Ball-Burnett, M. 1999a. 299 Serial effects of high-resistance and prolonged endurance training on $\mathrm{Na}+\mathrm{K}+$ pump 300 concentration and enzymatic activities in human vastus lateralis. Acta Physiol Scand 165: 301 177-184.

302 Green, H., Goreham, C., Ouyang, J., Ball-Burnett, M., and Ranney, D. 1999b. Regulation of 303 fiber size, oxidative potential, and capillarization in human muscle by resistance exercise. Am J 304 Physiol 276: R591- R596.

305 Hoshino, D., Yoshida, Y., Kitaoka, Y., Hatta, H., and Bonen, A. 2013. High-intensity interval 306 training increases intrinsic rates of mitochondrial fatty acid oxidation in rat red and white 307 skeletal muscle. Appl Physiol Nutr Metab 38: 326-333.

308 Iqbal, S., Ostojic, O., Singh, K., Joseph, A.M., and Hood, D.A. 2013. Expression of 309 mitochondrial fission and fusion regulatory proteins in skeletal muscle during chronic use and 310 disuse. Muscle Nerve 48: 963-970.

311 Ishihara, N., Fujita, Y., Oka, T., and Mihara, K. 2006. Regulation of mitochondrial morphology 312 through proteolytic cleavage of OPA1. EMBO J 25: 2966-2977.

313 Jamart, C., Naslain, D., Gilson, H., and Francaux, M. 2013. Higher activation of autophagy in 314 skeletal muscle of mice during endurance exercise in the fasted state. Am J Physiol Endocrinol 315 Metab 305: E964-E974.

316 Juel, C., Holten, M.K., and Dela, F. 2004. Effects of strength training on muscle lactate release 317 and MCT1 and MCT4 content in healthy and type 2 diabetic humans. J Physiol 556: 297-304.

318 Karlsson, H.K., Nilsson, P.A., Nilsson, J., Chibalin, A.V., Zierath, J.R., and Blomstrand, E. 2004.

319 Branched-chain amino acids increase p70S6k phosphorylation in human skeletal muscle after 320 resistance exercise. Am J Physiol Endocrinol Metab 287: E1-E7.

321 Kashatus, J.A., Nascimento, A., Myers, L.J., Sher, A., Byrne, F.L., Hoehn, K.L., et al. 2015. 322 Erk2 phosphorylation of Drp1 promotes mitochondrial fission and MAPK-driven tumor growth. 323 Mol Cell 57: 537-551.

324 Kitaoka, Y., Takahashi, Y., Machida, M., Takeda, K., Takemasa, T., and Hatta, H. 2014. Effect of 
325 AMPK activation on monocarboxylate transporter (MCT)1 and MCT4 in denervated muscle. J 326 Physiol Sci 64: 59-64.

327 Lira, V.A., Okutsu, M., Zhang, M., Greene, N.P., Laker, R.C., Breen, D.S., et al. 2013. 328 Autophagy is required for exercise training-induced skeletal muscle adaptation and 329 improvement of physical performance. FASEB J 27: 4184-4193.

330 Meeusen, S., DeVay, R., Block, J., Cassidy-Stone, A., Wayson, S., McCaffery, J.M., et al. 2006. 331 Mitochondrial inner-membrane fusion and crista maintenance requires the dynamin-related 332 GTPase Mgm1. Cell 127: 383-395.

333 Moore, D.R., Atherton, P.J., Rennie, M.J., Tarnopolsky, M.A., and Phillips, S.M. 2011. 334 Resistance exercise enhances mTOR and MAPK signalling in human muscle over that seen at 335 rest after bolus protein ingestion. Acta Physiol (Oxf) 201: 365-372.

336 Ogasawara, R., Kobayashi, K., Tsutaki, A., Lee, K., Abe, T., Fujita, S., et al. 2013. mTOR 337 signaling response to resistance exercise is altered by chronic resistance training and detraining 338 in skeletal muscle. J Appl Physiol (1985) 114: 934-940.

339 Ogasawara, R., Sato, K., Matsutani, K., Nakazato, K., and Fujita, S. 2014. The order of 340 concurrent endurance and resistance exercise modifies mTOR signaling and protein synthesis in 341 rat skeletal muscle. Am J Physiol Endocrinol Metab 306: E1155-E1162.

342 Ogborn, D.I., McKay, B.R., Crane, J.D., Safdar, A., Akhtar, M., Parise, G., et al. 2015. Effects 343 of age and unaccustomed resistance exercise on mitochondrial transcript and protein abundance 344 in skeletal muscle of men. Am J Physiol Regul Integr Comp Physiol: 308: R734-R741.

345 Parone, P.A., Da Cruz, S., Tondera, D., Mattenberger, Y., James, D.I., Maechler, P., et al. 2008. 346 Preventing mitochondrial fission impairs mitochondrial function and leads to loss of 347 mitochondrial DNA. PLoS One 3: e3257.

348 Perry, C.G., Heigenhauser, G.J., Bonen, A., and Spriet, L.L. 2008. High-intensity aerobic 349 interval training increases fat and carbohydrate metabolic capacities in human skeletal muscle.

350 Appl Physiol Nutr Metab 33: 1112-1123.

351 Perry, C.G., Lally, J., Holloway, G.P., Heigenhauser, G.J., Bonen, A., and Spriet, L.L. 2010.

352 Repeated transient mRNA bursts precede increases in transcriptional and mitochondrial proteins 353 during training in human skeletal muscle. J Physiol 588: 4795-4810.

354 Pesta, D., Hoppel, F., Macek, C., Messner, H., Faulhaber, M., Kobel, C., et al. 2011. Similar 355 qualitative and quantitative changes of mitochondrial respiration following strength and 356 endurance training in normoxia and hypoxia in sedentary humans. Am J Physiol Regul Integr 357 Comp Physiol 301: R1078-R1087. 
358 Picard, M., Azuelos, I., Jung, B., Giordano, C., Matecki, S., Hussain, S., et al. 2015. Mechanical 359 ventilation triggers abnormal mitochondrial dynamics and morphology in the diaphragm. $\mathbf{J}$ 360 Appl Physiol (1985) 118: 1161-1171.

361 Picard, M., Gentil, B.J., McManus, M.J., White, K., St Louis, K., Gartside, S.E., et al. 2013. 362 Acute exercise remodels mitochondrial membrane interactions in mouse skeletal muscle. J 363 Appl Physiol (1985) 115: 1562-1571.

364 Porter, C., Reidy, P.T., Bhattarai, N., Sidossis, L.S., and Rasmussen, B.B. 2014. Resistance 365 Exercise Training Alters Mitochondrial Function in Human Skeletal Muscle. Med Sci Sports 366 Exerc.

367 Romanello, V., Guadagnin, E., Gomes, L., Roder, I., Sandri, C., Petersen, Y., et al. 2010. 368 Mitochondrial fission and remodelling contributes to muscle atrophy. EMBO J 29: 1774-1785.

369 Salvadego, D., Domenis, R., Lazzer, S., Porcelli, S., Rittweger, J., Rizzo, G., et al. 2013. 370 Skeletal muscle oxidative function in vivo and ex vivo in athletes with marked hypertrophy 371 from resistance training. J Appl Physiol (1985) 114: 1527-1535.

372 Serasinghe, M.N., Wieder, S.Y., Renault, T.T., Elkholi, R., Asciolla, J.J., Yao, J.L., et al. 2015. 373 Mitochondrial division is requisite to RAS-induced transformation and targeted by oncogenic 374 MAPK pathway inhibitors. Mol Cell 57: 521-536.

375 Taguchi, N., Ishihara, N., Jofuku, A., Oka, T., and Mihara, K. 2007. Mitotic phosphorylation of 376 dynamin-related GTPase Drp1 participates in mitochondrial fission. J Biol Chem 282: 377 11521-11529.

378 Tesch, P.A., Thorsson, A., and Colliander, E.B. 1990. Effects of eccentric and concentric 379 resistance training on skeletal muscle substrates, enzyme activities and capillary supply. Acta 380 Physiol Scand 140: 575-580.

381 Wang, X., Li, H., Zheng, A., Yang, L., Liu, J., Chen, C., et al. 2014. Mitochondrial 382 dysfunction-associated OPA1 cleavage contributes to muscle degeneration: preventative effect 383 of hydroxytyrosol acetate. Cell Death Dis 5: e1521.

384

385

386

387

388 
391 Figure Legends.

392 Figure 1. Mitochondrial dynamics protein expression after acute resistance exercise in rat 393 skeletal muscle. (A) Mitochondrial fission proteins. (B) Mitochondrial fusion proteins. Data are

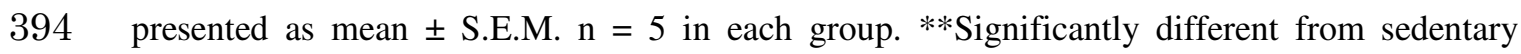
395 subjects $(P<0.01)$ and $*$ Significantly different from sedentary subjects $(P<0.05)$.

Figure 2. Mitochondrial dynamics protein expression after 4 weeks of resistance training in 400 rat skeletal muscle. Data are presented as mean \pm S.E.M. $\mathrm{n}=5$ in each group. ${ }^{* * \text { Significantly }}$ 401 different from control subjects $(P<0.01)$ and $*$ Significantly different from control subjects $402 \quad(P<0.05)$

Figure 3. Energy metabolism protein expression after 4 weeks of resistance training in rat 
$\begin{array}{cccccc}\text { SED } & \mathrm{RE} & \mathrm{RE}+1 \mathrm{~h} & \mathrm{RE}+3 \mathrm{~h} & \mathrm{RE}+6 \mathrm{~h} & \mathrm{RE}+24 \mathrm{~h} \\ \text { Applied Physiology, Nutrition, and Metabolism }\end{array}$
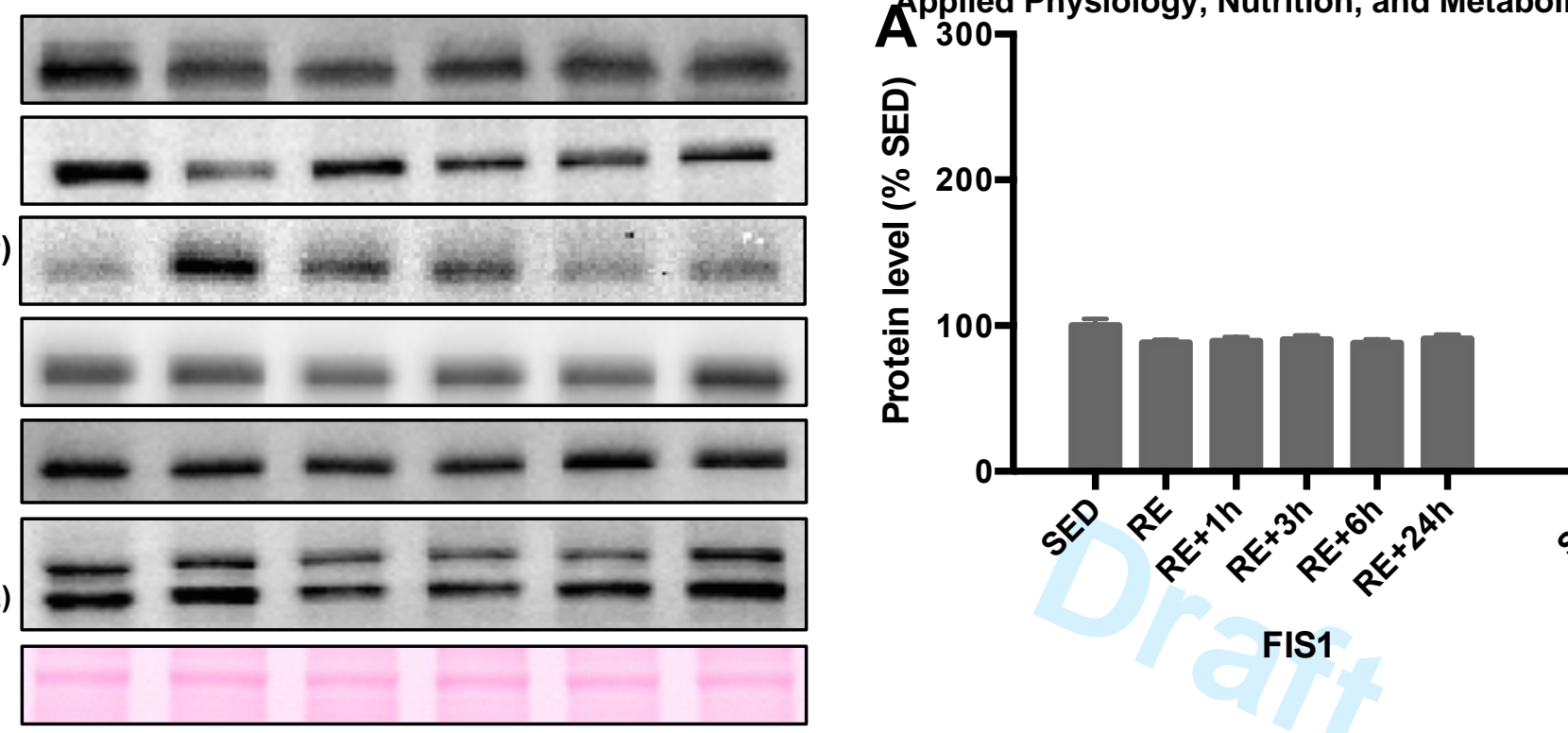

FIS1
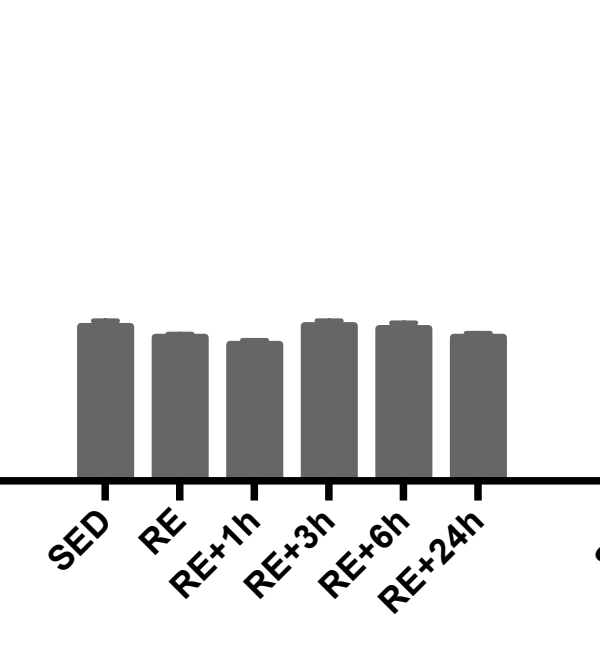

DRP1

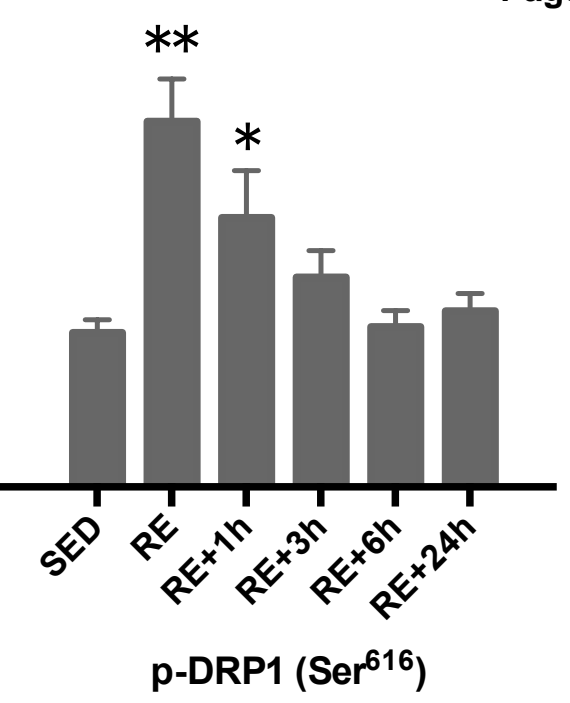

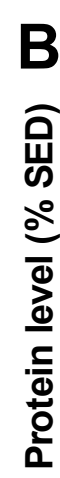

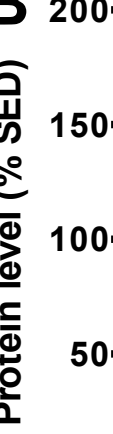
0

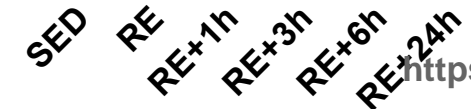

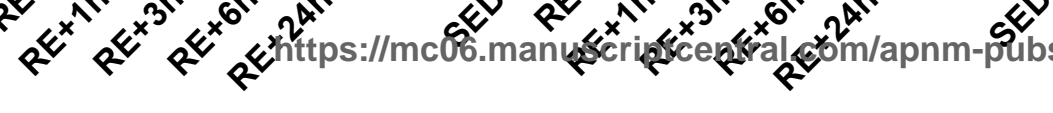

MFN2

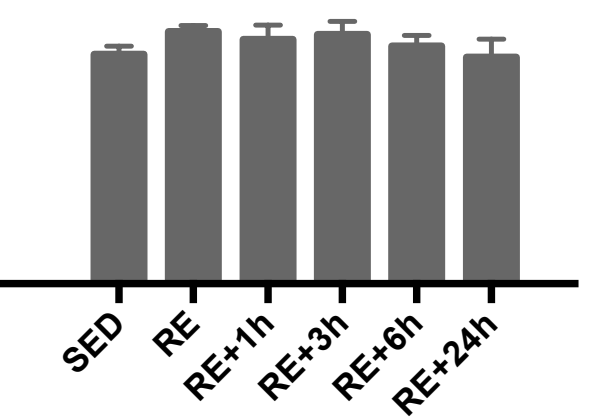

total OPA1

Page 16 of 18 


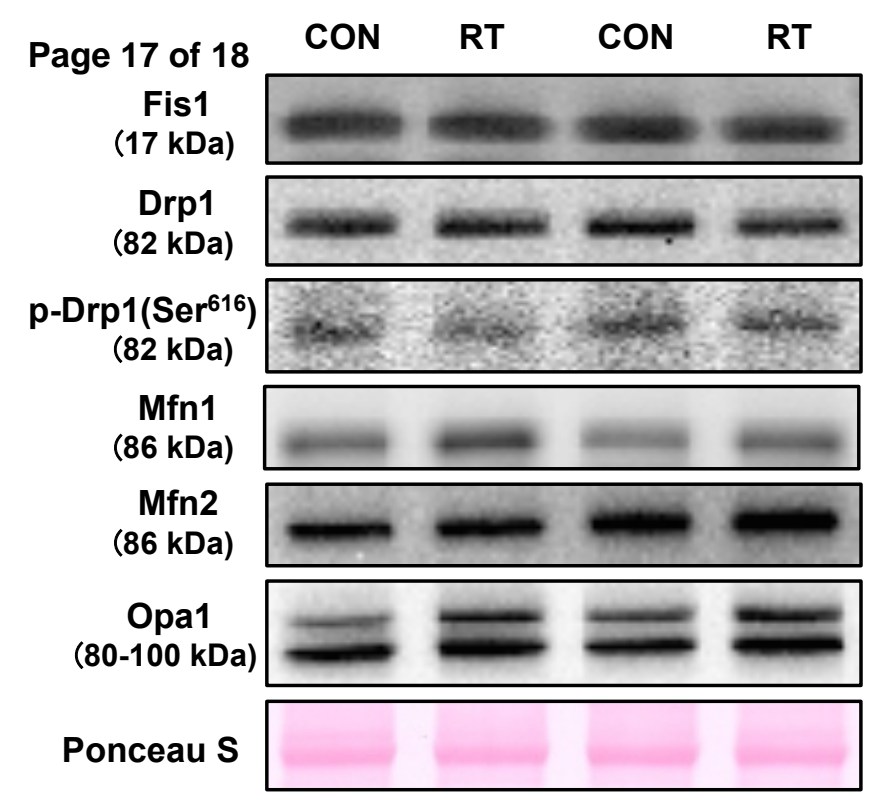

Applied Physiology, Nutrition, and Metabolism

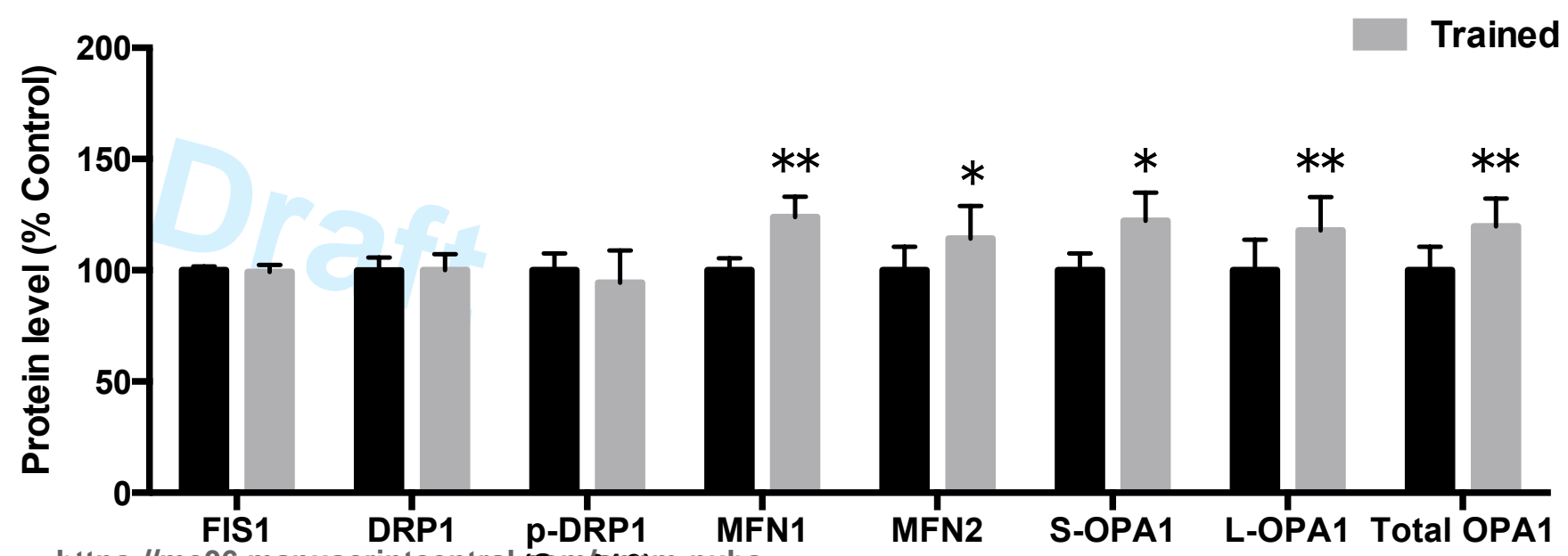

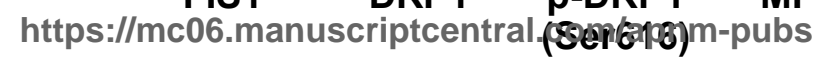




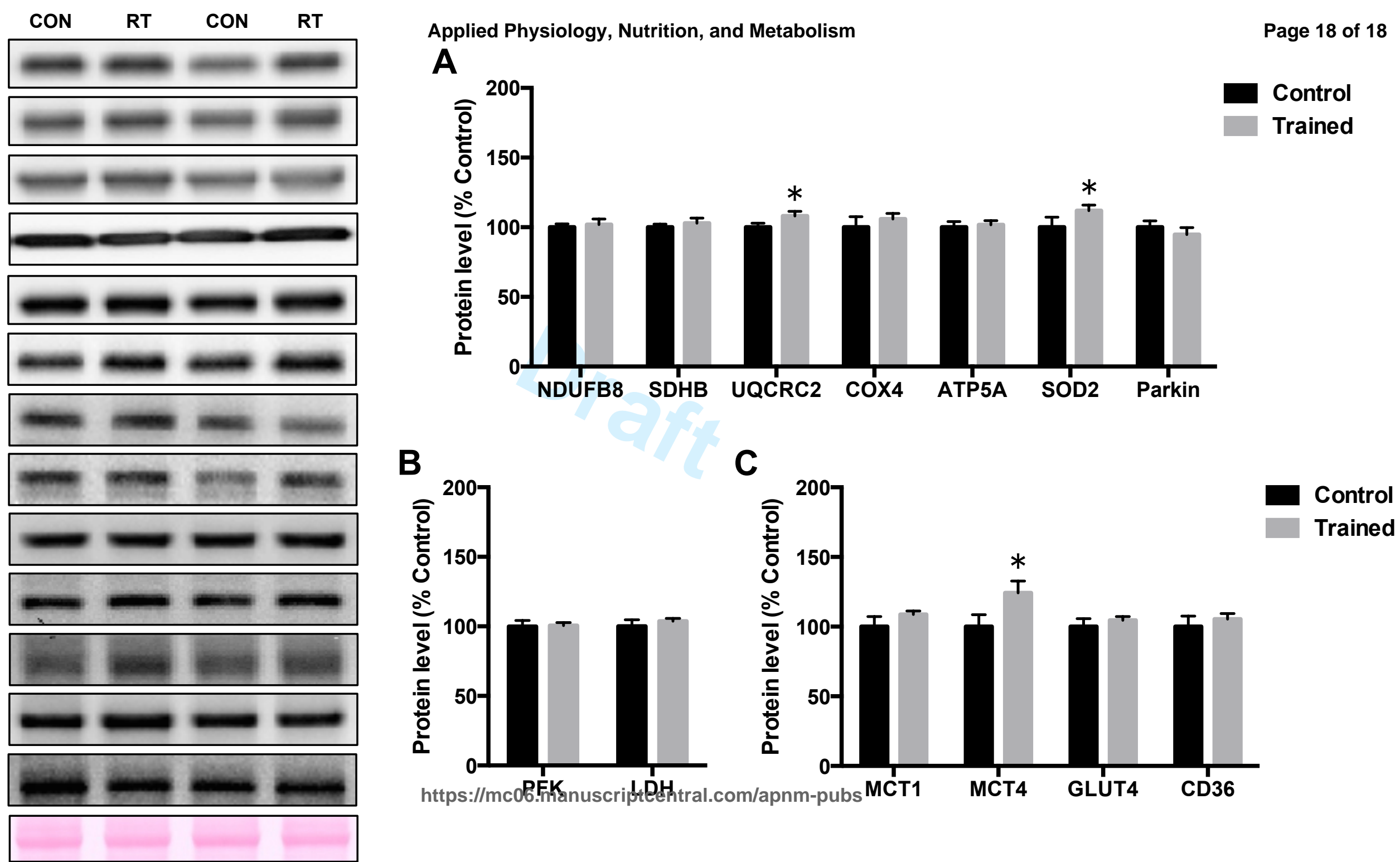

